\title{
A Special Research on Homo Cordial Labeling of Spider Graph
}

\author{
S.Sriram, R.Govindarajan
}

\begin{abstract}
Let $G=(V, E)$ be a graph with p vertices and $q$ edges. $A$ Homo Cordial Labeling of a graph $G$ with vertex set $V$ is a bijection from $V$ to $\{0,1\}$ such that each edge uv is assigned the label 1 if $f(u)=f(v)$ or 0 if $f(u) \neq f(v)$ with the condition that the number of vertices labelled with 0 and the number of vertices labelled with 1 differ by at most 1 and the number of edges labelled with 0 and the number of edges labelled with 1 differ by at most 1. The graph that admits a Homo-Cordial labelling is called Homo Cordial graph. In this paper we prove that the spider graph $S P\left(1^{m}, 2^{t}\right)$ is Homo-Cordial labelling graph and further study on the generalisation of labelling spider graph $\operatorname{SP}\left(1^{m}, 2^{t}\right)$
\end{abstract}

Keywords : Homo Cordial graphs, Homo Cordial labelling.

\section{INTRODUCTION}

A graph $\mathrm{G}$ is a finite nonempty set of objects called vertices and edges. All graphs considered here are finite, simple and undirected. Gallian[1] has given a dynamic survey of graph labelling. The origin of graph labelings can be attributed to Rosa. A Path related Homo Cordial graph was introduced by Dr.A.Nellai Murugan and A.Mathubala[2,3,4]. Motivated towards the labelling of homo cordial labelling of graphs In this paper we prove that spider graph $S P\left(1^{m}, 2^{t}\right)$.

is Homo Cordial labelling graph. Further to generalise the concept of homo cordial labelling of spider graph $S P\left(1^{m}, 2^{t}\right)$ we have ascertained the ways in which the number of labels assigned with 0 and number of labels assigned with 1 so as to identify the phenomena of spider graph $S P\left(1^{m}, 2^{t}\right)$ to be called a homo cordial labelling graph.

\section{PRELIMINARIES}

Definition 2.1: A tree is called a spider if it has a centre vertex $\mathrm{C}$ of degree $\mathrm{R}>1$ and all the other vertex is either a leaf or with degree 2. Thus a spider is an amalgamation of $\mathrm{k}$ paths with various lengths. If it has $X_{1}{ }^{\prime} s$ of length ${ }^{a_{1}}$,

Revised Manuscript Received on July 08, 2019.

S.Sriram, Assistant Professor, Department of Mathematics, Patrician College of Arts and Science, Adyar,Chennai-20, India.

Dr.R.Govindarajan, Associate Professor and Head (Retd.), P.G and U.G, Department of Mathematics, D.G.Vaishnav College, Arumbakkam, Chennai,India
$X_{2}{ }^{\prime} s$ paths of length ${ }^{a_{2}}$ etc. We shall denote the spider by $S P\left(a_{1}^{x_{1}}, a_{2}^{x_{2}}, a_{3}^{x_{3}} \ldots a_{m}^{x_{m}}\right)$ where $a_{1} \mathrm{p} a_{2} \mathrm{p} a_{3} \mathrm{p} \ldots a_{m}$ and $x_{1}+x_{2}+\ldots+x_{m}=R$

\section{MaIn Results}

Theorem .3.1 : The Spider graph $S P\left(1^{m}, 2^{t}\right)$ is a homo cordial labelling graph

Proof: Let $G=\operatorname{SP}\left(1^{m}, 2^{t}\right)$ be a Spider graph.

We know that a tree is called a spider if it has a centre vertex $C$ of degree $R>1$ and all the other vertex is either a leaf or with degree 2. Thus a spider is an amalgamation of $\mathrm{k}$ paths with various lengths. If it has $X_{1}{ }^{\prime} s$ of length ${ }^{a_{1}}$, $X_{2}{ }^{\prime} s$ paths of length ${ }^{a_{2}}$ etc. We shall denote the spider by $S P\left(a_{1}^{x_{1}}, a_{2}^{x_{2}}, a_{3}^{x_{3}} \ldots a_{m}^{x_{m}}\right)$ where $a_{1} \mathrm{p} a_{2} \mathrm{p} a_{3} \mathrm{p} \ldots a_{m}$ and $x_{1}+x_{2}+\ldots+x_{m}=R$ the

Vertex set

$V\left(S P\left(1^{m}, 2^{t}\right)\right)=\left\{u, v_{i}, u_{j}: 1 \leq i \leq m, 1 \leq j \leq 2 t\right\}$

and edge set

$E\left(S P\left(1^{m}, 2^{t}\right)\right)=\left\{e_{i}=u v_{i}: 1 \leq i \leq m, e_{i}^{\prime}=u u_{j:} 1 \leq j \leq t, e^{\prime \prime}{ }_{i}=u_{j} u_{t+j}: 1 \leq j \leq t\right\}$

Now to label the vertices let us consider the bijective function $f: V \rightarrow\{0,1\}$ such that such that each edge uv is assigned the label 1 if $\mathrm{f}(\mathrm{u})=\mathrm{f}(\mathrm{v})$ or 0 if $f(u) \neq f(v)$ with the condition that the number of vertices labelled with 0 and the number of vertices labelled with 1 differ by at most 1 and the number of edges labelled with 0 and the number of edges labelled with 1 differ by at most 1 . We define the labelling of vertices as follows

$$
\begin{aligned}
& f(u)=0 f\left(v_{i}\right)=1_{\text {for }} i \equiv 1(\bmod m) \\
& f\left(v_{i}\right)=0 \text { for } i \equiv 0(\bmod m) \text { where } 1 \leq i \leq m \\
& f\left(u_{j}\right)=1 \text { for } j \equiv 1(\bmod t) \\
& f\left(u_{j}\right)=0 \text { for } j \equiv 0(\bmod t) \text { where } 1 \leq j \leq 2 t
\end{aligned}
$$

Then the induced edge labelling for the graph 


\section{A Special Research on Homo Cordial Labeling of Spider Graph}

$$
\begin{aligned}
& G=S P\left(1^{m}, 2^{t}\right) \text { are } \\
& f^{*}\left(u v_{i}\right)=0 \text { for } i \equiv 1(\bmod m) \\
& f^{*}\left(u v_{i}\right)=1 \text { for } i \equiv 0(\bmod m) \\
& f^{*}\left(u u_{j}\right)=1 \\
& f^{*}\left(u_{j} u_{t+j}\right)=0 \text { where } 1 \leq j \leq 2 t
\end{aligned}
$$

Noticing the induced edge labelling we find that the number of vertices labelled with 0 is $\mathrm{n}$ and the number of vertices labelled with 1 is $\mathrm{n}$ and that the number of edges labelled with 0 is $n+1$ and the number of edges labelled with 1 is $n$. Hence $\left|v_{f}(0)-v_{f}(1)\right| \leq 1$ and $\left|e_{f}(0)-e_{f}(1)\right| \leq 1$. Therefore the Spider graph $G=S P\left(1^{m}, 2^{t}\right)$ is a homo cordial labelling graph.

Example 3.2 : Consider the Spider graph $S P\left(1^{2}, 2^{2}\right)$

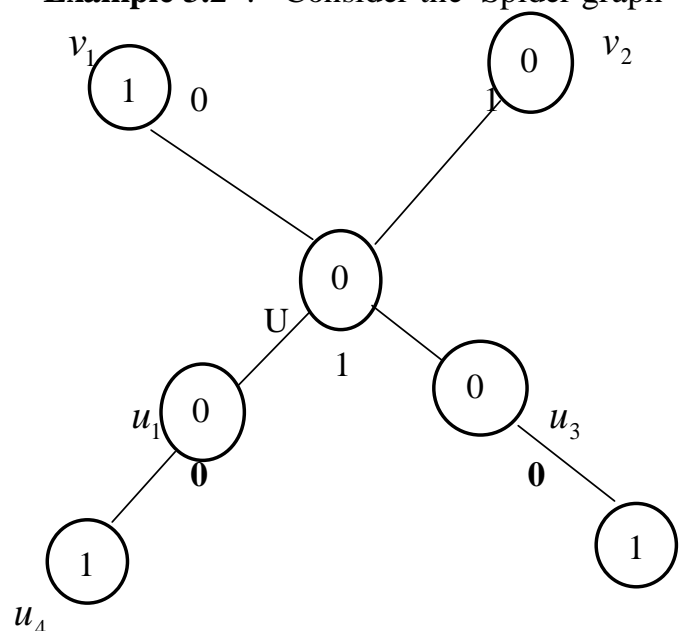

In order to generalise the Spider graph $S P\left(1^{m}, 2^{t}\right)$ for the various types we classify according to the values of $\mathrm{m}$ and $\mathrm{t}$ as follows
Case.(i) $m \mathrm{pt}$
Case.(ii) $m=t$
Case.(iii) $m \mathrm{f} t$

Where we define a relation between the possibilities of $1^{m}$ with that of $2^{t}$ as

$$
A_{1}=\{1,2,3 \ldots m\} \text {, where } A_{1} \text { is the possibilities of } 1^{m}
$$
and $A_{2}=\{1,2, \ldots t\}$, where $A_{2}$ is the possibilities of $2^{t}$ then the relation $R=A_{1} x A_{2}$ is defined as either $\mathrm{p}$, $=, \mathrm{f}$ as in Case.(i), 'Case.(ii) and Case.(iii) respectively.

Suppose for $\mathrm{m}=2$ and $\mathrm{t}=3$ we have $A_{1}=\{1,2\}$ and $A_{2}=\{1,2,3\}$
$R=A_{1} x A_{2}=\{(1,1)(1,2)(1,3)(2,2)(2,3)(2,1)\}$ we

identify that $(1,2)(1,3)(2,3)$ belongs to Case.(i) $(1,1)(2,2)$ belongs to Case. (ii) and $(2,1)$ belongs to Case.(iii) giving rise to the number of elements in the relation $\mathrm{R}$ and for $\mathrm{m}=2$ and $\mathrm{t}=3$ we have total number of elements $=2 \times 3=6$ and hence in general mxn elements for a spider graph $S P\left(1^{m}, 2^{t}\right)$

Definition 3.3 : For a Spider graph $S P\left(1^{m}, 2^{t}\right)$ let us define a relation $\mathrm{R}$ from ${ }^{A_{1}}$ to $A_{2}$ defined as a subset of the Cartesian product $A_{1} X A_{2}$ such that $A_{1}=\{x: 1 \leq x \leq m\}$ and $A_{2}=\{y: 1 \leq y \leq t\}$.

The number of vertices among each of the possibilities of $1^{m}$ is 1 excluding the centre vertex and the number of edges contributed for each of the possibilities of $1^{m}$ is 1 . Also the number of vertices among each of the possibilities of $2^{t}$ is 2 excluding the centre vertex and the number of edges contributed for each of the possibilities of $2^{t}$ is 2 .

Definition 3.4: Basic Spider graph $S P\left(1^{m}, 2^{t}\right)$ for $\mathrm{m}=1$ and $\mathrm{t}=1$. The Spider graph $\operatorname{SP}\left(1^{1}, 2^{1}\right)$ is defined to have the vertex set $V\left(S P\left(1^{1}, 2^{1}\right)\right)=\left\{u, u_{1}, v_{1}, v_{2}\right\}$ and $E\left(S P\left(1^{1}, 2^{1}\right)\right)=\left\{u u_{1}, u v_{1}, v_{1} v_{2}\right\}$ with number of vertices of $S P\left(1^{m}, 2^{t}\right)=4$ and number of edges $S P\left(1^{m}, 2^{t}\right)=3$.

Note. 1 : For each possibility of $1^{m}$ the number of vertices increases by 1 and edges increase by 1 and for each possibility of $2^{t}$ the number of vertices increases by 2 and edges increases by 2 .

Note.2: By Labelling procedure adopted in Theorem.3.1 to prove Spider graph $S P\left(1^{m}, 2^{t}\right)$ is homo cordial graph we notice that the basic Spider graph $S P\left(1^{m}, 2^{t}\right)$ for $\mathrm{m}=1$ and $\mathrm{t}=1$ has 4 labels (both vertices and edges ) assigned with 0 and 3 labels(both vertices and edges ) assigned with 1.

Definition 3.5: One part of Spider graph $S P\left(1^{m}, 2^{t}\right)$ : For a Spider graph $S P\left(1^{m}, 2^{t}\right)$ from the basic Spider graph $S P\left(1^{1}, 2^{1}\right)$ we add one part denoted by $T\left(F_{1}\right)$ and the resulting Spider graph is 
$\operatorname{SP}\left(1^{2}, 2^{1}\right)$. The resulting graph $S P\left(1^{2}, 2^{1}\right)$ is said to increase the number of labels assigned as 0 by 1 and the number of labels assigned as 1 by 1 to the existing basic Spider graph $S P\left(1^{1}, 2^{1}\right)$ For a Spider graph $S P\left(1^{m}, 2^{t}\right)$ from the basic Spider graph $S P\left(1^{1}, 2^{1}\right)$ we add one part denoted by $T\left(F_{2}\right)$ and the resulting Spider graph is $S P\left(1^{1}, 2^{2}\right)$. The resulting graph $\operatorname{SP}\left(1^{1}, 2^{2}\right)$ is said to increase the number of labels assigned as 0 by 2 and the number of labels assigned as 1 by 2 to the existing basic Spider graph $S P\left(1^{1}, 2^{1}\right)$

Note.1: We denote the number of labels assigned with 0 (both vertices and edges)to the Spider graph $S P\left(1^{m}, 2^{t}\right)$ by $T_{0}\left(S P\left(1^{m}, 2^{t}\right)\right)$ and the number of labels assigned with 1(both vertices and edges) by $T_{1}\left(\operatorname{SP}\left(1^{m}, 2^{t}\right)\right)$

Theorem:3.7: If Spider graph $S P\left(1^{m}, 2^{t}\right)$ is homo cordial graph then $T_{0}\left(S P\left(1^{m}, 2^{1}\right)\right)=T_{0}\left(S P\left(1^{1}, 2^{1}\right)\right)+(m-1) T_{0}\left(F_{1}\right)$ $T_{1}\left(S P\left(1^{m}, 2^{1}\right)\right)=T_{1}\left(S P\left(1^{1}, 2^{1}\right)\right)+(m-1) T_{1}\left(F_{1}\right)$ and

Proof: Consider the Spider graph $S P\left(1^{m}, 2^{t}\right)$ which is proved as homo cordial graph as in Theorem.3.1. Let us prove the theorem by method of Mathematical induction on $\mathrm{m}$, where $\mathrm{m}$ is the number of paths from the centre vertex.

Let us prove for the $1^{\text {st }}$ positive integer $\mathrm{m}$ i.e $\mathrm{m}=2$

We have $S P\left(1^{1}, 2^{1}\right)$ which is the basic Spider graph and contains labelling of 40 's (both vertices and edges) and 3 1's (both vertices and edges). On adding one part $T\left(F_{1}\right)$ we find that there is an increase in label assigned with 0 by 1 and label assigned with 1 by 1 . Hence $S P\left(1^{2}, 2^{2}\right)$ has 5 0 's and 41 's. Hence

$$
\begin{aligned}
& T_{0}\left(\operatorname{SP}\left(1^{2}, 2^{1}\right)\right)=T_{0}\left(\operatorname{SP}\left(1^{1}, 2^{1}\right)\right)+(2-1) T_{0}\left(F_{1}\right) \\
& T_{0}\left(\operatorname{SP}\left(1^{2}, 2^{1}\right)\right)=T_{0}\left(\operatorname{SP}\left(1^{1}, 2^{1}\right)\right)+T_{0}\left(F_{1}\right) \\
& T_{0}\left(\operatorname{SP}\left(1^{2}, 2^{1}\right)\right)=4+1=5
\end{aligned}
$$

Similarly
Definition 3.6: One part of Spider graph $S P\left(1^{m}, 2^{t}\right)$ :

$$
\begin{aligned}
T_{1}\left(S P\left(1^{m}, 2^{1}\right)\right) & =T_{1}\left(S P\left(1^{1}, 2^{1}\right)\right)+(m-1) T_{1}\left(F_{1}\right) \\
T_{1}\left(S P\left(1^{2}, 2^{1}\right)\right) & =T_{1}\left(S P\left(1^{1}, 2^{1}\right)\right)+(2-1) T_{1}\left(F_{1}\right) \\
T_{1}\left(S P\left(1^{2}, 2^{1}\right)\right) & =T_{1}\left(S P\left(1^{1}, 2^{1}\right)\right)+T_{1}\left(F_{1}\right) \\
T_{1}\left(S P\left(1^{2}, 2^{1}\right)\right) & =3+1=4
\end{aligned}
$$

Hence it is true for $m=2$.

Now let us assume that the result is true for $\mathrm{m}=\mathrm{k}$

$$
\begin{aligned}
& T_{0}\left(S P\left(1^{k}, 2^{1}\right)\right)=T_{0}\left(S P\left(1^{1}, 2^{1}\right)\right)+(k-1) T_{0}\left(F_{1}\right) \\
& T_{1}\left(S P\left(1^{k}, 2^{1}\right)\right)=T_{1}\left(S P\left(1^{1}, 2^{1}\right)\right)+(k-1) T_{1}\left(F_{1}\right)
\end{aligned}
$$

Now let us prove for $\mathrm{m}=\mathrm{k}+1$

i.e to Prove

$$
\begin{aligned}
& T_{0}\left(S P\left(1^{k+1}, 2^{1}\right)\right)=T_{0}\left(S P\left(1^{1}, 2^{1}\right)\right)+(k) T_{0}\left(F_{1}\right) \\
& T_{1}\left(S P\left(1^{k+1}, 2^{1}\right)\right)=T_{1}\left(S P\left(1^{1}, 2^{1}\right)\right)+(k) T_{1}\left(F_{1}\right) \\
& \text { Consider }^{T_{0}\left(S P\left(1^{k+1}, 2^{1}\right)\right)}=T_{0}\left(S P\left(1^{k}, 2^{1}\right)\right)+T_{0}\left(F_{1}\right) \\
& T_{0}\left(S P\left(1^{k+1}, 2^{1}\right)\right)=T_{0}\left(S P\left(1^{1}, 2^{1}\right)\right)+(k-1) T_{0}\left(F_{1}\right)+T_{0}\left(F_{1}\right)
\end{aligned}
$$

Hence

$T_{0}\left(S P\left(1^{k+1}, 2^{1}\right)\right)=T_{0}\left(S P\left(1^{1}, 2^{1}\right)\right)+(k) T_{0}\left(F_{1}\right)$

$$
\begin{array}{ccc}
\text { Similarly } & \text { we } \quad \begin{array}{c}
\text { can } \\
T_{1}\left(\operatorname{SP}\left(1^{k+1}, 2^{1}\right)\right)=T_{1}\left(\operatorname{SP}\left(1^{1}, 2^{1}\right)\right)+(k) T_{1}\left(F_{1}\right)
\end{array} .
\end{array}
$$

Hence the theorem by induction.

Corollary 3.8 : If for Spider graph $\operatorname{SP}\left(1^{m}, 2^{1}\right)$ which is homo cordial labelling graph removal of each part $T\left(F_{1}\right)$ reduces the total number of vertices and edges labelled with 0 by 1 and total number of vertices and edges labelled with 1 by 1 and reduces to the basic Spider graph $\operatorname{SP}\left(1^{1}, 2^{1}\right)$

Proof: Consider the Spider graph $S P\left(1^{m}, 2^{1}\right)$ which is homo cordial labelling graph . Now we remove one part $T\left(F_{1}\right)$ and one part of $T\left(F_{1}\right)$ consists of total number of vertices and edges labelled with 0 as 1 and total number of vertices and edges labelled with 1 as 1 . Continuing this procedure m-1 times we can reduce the Spider graph $S P\left(1^{m}, 2^{1}\right)$ to the basic Spider graph $S P\left(1^{1}, 2^{1}\right)$. Hence the proof.

Theorem:3.9: If Spider graph $\operatorname{SP}\left(1^{m}, 2^{t}\right)$ is homo cordial graph then 


\section{A Special Research on Homo Cordial Labeling of Spider Graph}

$$
\begin{aligned}
& T_{0}\left(S P\left(1^{1}, 2^{t}\right)\right)=T_{0}\left(S P\left(1^{1}, 2^{1}\right)\right)+(t-1) T_{0}\left(F_{2}\right) \\
& T_{1}\left(S P\left(1^{1}, 2^{t}\right)\right)=T_{0}\left(S P\left(1^{1}, 2^{1}\right)\right)+(t-1) T_{0}\left(F_{2}\right)
\end{aligned}
$$

Proof: Consider the Spider graph $\operatorname{SP}\left(1^{m}, 2^{t}\right)$ which is proved as homo cordial graph as in Theorem.3.1. Let us prove the theorem by method of Mathematical induction on $t$, where $t$ is the number of paths from the centre vertex.

Let us prove for the $1^{\text {st }}$ positive integer $\mathrm{t}$ i.e $\mathrm{t}=2$

We have $\operatorname{SP}\left(1^{1}, 2^{1}\right)$ which is the basic Spider graph and contains labelling of 40 's (both vertices and edges) and 3 1's (both vertices and edges). On adding one part $T\left(F_{2}\right)$ we find that there is an increase in label assigned with 0 by 2 and label assigned with 1 by 2 . Hence $S P\left(1^{2}, 2^{2}\right)$ has 6 0 's and 51 's. Hence

$$
\begin{aligned}
& T_{0}\left(S P\left(1^{1}, 2^{2}\right)\right)=T_{0}\left(S P\left(1^{1}, 2^{1}\right)\right)+(2-1) T_{0}\left(F_{2}\right) \\
& T_{0}\left(S P\left(1^{1}, 2^{2}\right)\right)=T_{0}\left(S P\left(1^{1}, 2^{1}\right)\right)+T_{0}\left(F_{2}\right) \\
& T_{0}\left(S P\left(1^{1}, 2^{2}\right)\right)=4+2=6
\end{aligned}
$$

Similarly

$$
\begin{gathered}
T_{1}\left(S P\left(1^{1}, 2^{t}\right)\right)=T_{1}\left(S P\left(1^{1}, 2^{1}\right)\right)+(t-1) T_{1}\left(F_{2}\right) \\
T_{1}\left(S P\left(1^{1}, 2^{2}\right)\right)=T_{1}\left(S P\left(1^{1}, 2^{1}\right)\right)+(2-1) T_{1}\left(F_{2}\right) \\
T_{1}\left(S P\left(1^{1}, 2^{2}\right)\right)=T_{1}\left(S P\left(1^{1}, 2^{1}\right)\right)+T_{1}\left(F_{2}\right) \\
T_{1}\left(S P\left(1^{1}, 2^{2}\right)\right)=3+2=5
\end{gathered}
$$

Hence it is true for $m=2$.

Now let us assume that the result is true for $\mathrm{t}=\mathrm{k}$

$$
\begin{aligned}
& T_{0}\left(S P\left(1^{1}, 2^{k}\right)\right)=T_{0}\left(S P\left(1^{1}, 2^{1}\right)\right)+(k-1) T_{0}\left(F_{2}\right. \\
& T_{1}\left(S P\left(1^{1}, 2^{k}\right)\right)=T_{1}\left(S P\left(1^{1}, 2^{1}\right)\right)+(k-1) T_{1}\left(F_{2}\right)
\end{aligned}
$$

Now let us prove for $\mathrm{m}=\mathrm{k}+1$ i.e to Prove

$$
\begin{gathered}
T_{0}\left(S P\left(1^{1}, 2^{k+1}\right)\right)=T_{0}\left(S P\left(1^{1}, 2^{1}\right)\right)+(k) T_{0}\left(F_{2}\right) \\
T_{1}\left(S P\left(1^{1}, 2^{k+1}\right)\right)=T_{1}\left(S P\left(1^{1}, 2^{1}\right)\right)+(k) T_{1}\left(F_{2}\right)
\end{gathered}
$$

Consider

$$
\begin{aligned}
& T_{0}\left(S P\left(1^{1}, 2^{k+1}\right)\right)=T_{0}\left(S P\left(1^{1}, 2^{1}\right)\right)+(k-1) T_{0}\left(F_{2}\right)+T_{0}\left(F_{2}\right) \\
& T_{0}\left(S P\left(1^{1}, 2^{k+1}\right)\right)=T_{0}\left(S P\left(1^{1}, 2^{1}\right)\right)+k T_{0}\left(F_{2}\right)
\end{aligned}
$$

Hence

$$
T_{0}\left(S P\left(1^{k+1}, 2^{1}\right)\right)=T_{0}\left(S P\left(1^{1}, 2^{1}\right)\right)+(k) T_{0}\left(F_{1}\right)
$$

$$
\text { Similarly we can prove }
$$$$
T_{1}\left(S P\left(1^{1}, 2^{k+1}\right)\right)=T_{1}\left(S P\left(1^{1}, 2^{1}\right)\right)+k T_{1}\left(F_{2}\right)
$$

Hence the theorem by induction.
Corollary 3.10 : If for Spider graph $S P\left(1^{1}, 2^{t}\right)$ which is homo cordial labelling graph removal of each part $T\left(F_{2}\right)$ reduces the total number of vertices and edges labelled with 0 by 2 and total number of vertices and edges labelled with 1 by 2 and reduces to the basic Spider graph $\operatorname{SP}\left(1^{1}, 2^{1}\right)$

Proof: Consider the Spider graph $\operatorname{SP}\left(1^{1}, 2^{t}\right)$ which is homo cordial labelling graph . Now we remove one part $T\left(F_{2}\right)$ and one part of $T\left(F_{2}\right)$ consists of total number of vertices and edges labelled with 0 as 2 and total number of vertices and edges labelled with 1 as 2 . Continuing this procedure $\mathrm{t}-1$ times we can reduce the Spider graph $S P\left(1^{1}, 2^{t}\right)$ to the basic Spider graph $S P\left(1^{1}, 2^{1}\right)$. Hence the proof.

Theorem:3.11: If Spider graph $S P\left(1^{m}, 2^{t}\right)$ is homo cordial graph then

$$
\begin{aligned}
& T_{0}\left(S P\left(1^{m}, 2^{t}\right)\right)=2 T_{0}\left(S P\left(1^{1}, 2^{1}\right)\right)+(m-1) T_{0}\left(F_{1}\right)+(t-1) T_{0}\left(F_{2}\right) \\
& T_{1}\left(S P\left(1^{m}, 2^{t}\right)\right)=2 T_{1}\left(\operatorname{SP}\left(1^{1}, 2^{1}\right)\right)+(m-1) T_{1}\left(F_{1}\right)+(t-1) T_{0}\left(F_{2}\right)
\end{aligned}
$$

Proof: Given the Spider graph $S P\left(1^{m}, 2^{t}\right)$ is a homo cordial graph. Now from theorem $3.7 \&$ theorem .3 .8 we find the following results

$$
\begin{aligned}
& T_{0}\left(S P\left(1^{m}, 2^{1}\right)\right)=T_{0}\left(S P\left(1^{1}, 2^{1}\right)\right)+(m-1) T_{0}\left(F_{1}\right) \\
& T_{1}\left(\operatorname{SP}\left(1^{m}, 2^{1}\right)\right)=T_{1}\left(S P\left(1^{1}, 2^{1}\right)\right)+(m-1) T_{1}\left(F_{1}\right) \\
& T_{0}\left(\operatorname{SP}\left(1^{1}, 2^{t}\right)\right)=T_{0}\left(\operatorname{SP}\left(1^{1}, 2^{1}\right)\right)+(t-1) T_{0}\left(F_{2}\right) \\
& T_{1}\left(\operatorname{SP}\left(1^{1}, 2^{t}\right)\right)=T_{0}\left(\operatorname{SP}\left(1^{1}, 2^{1}\right)\right)+(t-1) T_{0}\left(F_{2}\right)
\end{aligned}
$$

Now Consider

$$
T_{0}\left(S P\left(1^{m}, 2^{t}\right)\right)=T_{0}\left(S P\left(1^{m}, 2^{1}\right)\right)+T_{0}\left(S P\left(1^{1}, 2^{t}\right)\right)
$$

Which on substituting from the result

$T_{0}\left(S P\left(1^{\prime \prime}, 2^{\prime}\right)\right)=T_{0}\left(S P\left(1^{\prime}, 2^{\prime}\right)\right)+(m-1) T_{0}\left(F_{1}\right)+T_{0}\left(\operatorname{SP}\left(1^{\prime}, 2^{\prime}\right)\right)+T_{0}\left(S P\left(1^{\prime}, 2^{\prime}\right)\right)+(t-1) T_{0}\left(F_{2}\right)$

Simplifying we have

$T_{0}\left(S P\left(1^{m}, 2^{t}\right)\right)=2 T_{0}\left(S P\left(1^{1}, 2^{1}\right)\right)+(m-1) T_{0}\left(F_{1}\right)+(t-1) T_{0}\left(F_{2}\right)$

Similarly consider

$T_{1}\left(S P\left(1^{m}, 2^{t}\right)\right)=T_{1}\left(S P\left(1^{m}, 2^{1}\right)\right)+T_{1}\left(S P\left(1^{1}, 2^{t}\right)\right)$

Which on substituting from the result

$$
T_{1}\left(S P\left(1^{m}, 2^{t}\right)\right)=T_{1}\left(S P\left(1^{1}, 2^{1}\right)\right)+(m-1) T_{1}\left(F_{1}\right)+T_{1}\left(S P \left(1^{1}, 2\right.\right.
$$

Simplifying we have 


$$
T_{1}\left(S P\left(1^{m}, 2^{t}\right)\right)=2 T_{1}\left(S P\left(1^{1}, 2^{1}\right)\right)+(m-1) T_{1}\left(F_{1}\right)+(t-1) T_{(m-1)}\left(F_{-1}\right) \text { times we get the Spider graph } S P\left(1^{m}, 2^{t}\right) \text { with }
$$

Hence the proof. the labelling as specified by Theorem.3.1. Hence the proof..

Corollary 3.12 : If for Spider graph $S P\left(1^{m}, 2^{t}\right)$ which is homo cordial labelling graph removal of each part $T(F)=T\left(F_{1}\right)+T\left(F_{2}\right)$ reduces the total number of vertices and edges labelled with 0 by 3 and total number of vertices and edges labelled with 1 by 3 and reduces to the basic Spider graph $S P\left(1^{1}, 2^{1}\right)$

Proof: Consider the Spider graph $S P\left(1^{m}, 2^{t}\right)$ which is homo cordial labelling graph . Now we remove one part 1 . $T(F)=T\left(F_{1}\right)+T\left(F_{2}\right) T\left(F_{2}\right)$ which consists of total 2 . number of vertices and edges labelled with 0 as 3 and total number of vertices and edges labelled with 1 as 3.3 . Continuing this procedure $(\mathrm{m}-1)(\mathrm{t}-1)$ times we can reduce the Spider graph $S P\left(1^{m}, 2^{t}\right)$ to the basic Spider graph $S P\left(1^{1}, 2^{1}\right)$. Hence the proof.

Theorem:3.13: If G is a Spider graph $S P\left(1^{m}, 2^{t}\right)$ then the following are equivalent

(a) Spider graph $S P\left(1^{m}, 2^{t}\right)$ is homo cordial graph

(b) $T_{0}\left(S P\left(1^{m}, 2^{t}\right)\right)=2 T_{0}\left(S P\left(1^{1}, 2^{1}\right)\right)+(m-1) T_{0}\left(F_{1}\right)+(t-1) T_{0}\left(F_{2}\right)$ and

$T_{1}\left(S P\left(1^{m}, 2^{t}\right)\right)=2 T_{1}\left(S P\left(1^{1}, 2^{1}\right)\right)+(m-1) T_{1}\left(F_{1}\right)+(t-1) T_{0}\left(F_{2}\right)$

(c) Each part $T(F)$ of Spider graph $S P\left(1^{m}, 2^{t}\right)$ has 3 0 's and 31 's

Proof: Consider the graph G, the Spider graph $\operatorname{SP}\left(1^{m}, 2^{t}\right)$ claim

. To prove that the following are equivalent we
(a) Implies (b)
(b) Implies (c)
(c) Implies (a)

For (a) implies (b)

By considering the Theorem.3.1 and the labelling procedure we can claim the results in Theorem.3.11 and hence (b) is true

For (b) implies (c)

By considering the result obtained in theorem 3.11 and from the corollary 3.12 we are done.

For (c) implies (a)

By considering (c) we can obtain by adding each part $T(F)$ to the basic Spider graph $\operatorname{SP}\left(1^{1}, 2^{1}\right)$ consecutively

\section{CONCLUSION}

In this paper we have considered Spider Graph $S P\left(1^{m}, 2^{t}\right)$

and proved that it is homo cordial labelling graph and have identified a generalisation method for Spider graph. We are investigating on the other types of Spider graphs which can be also labelled so as to prove that they are homo cordial labelling graph.

\section{REFERENCES}

J.A . Gallian, A Dynamic Survey of Graph Labeling, Twentieth edition 2017

A.Nellai Murugan and A.Mathubala, Path Related Homo Cordial Graph, International Journal and Innovative Science Engineering and Technology. Vol.2, Issue 8, August 2015

A.Nellai Murugan and A.Mathubala, Cycle related Homo Cordial Graph, International Journal of Multidisciplinary Research and Devlopment, Vol.2, Issue 10 84-88, October 2015

4. A.Nellai Murugan and A.Mathubala, Special Class of Homo-Cordial Graphs, International Journal Emerging Technologies in Engineering Research, ISSN 2524-6410, Vol.2, Issue 3, October 2015, PP 1-5.

5. N.B. Rathod and K.K.Kanani, K-kodial labelling of Triangular Belt, Alternate Triangular Belt, Braid Graph and Z- International Journal of Mathematics and its application, Volume 5, Issue 4-E(2017),655-662

6. J.Gross and J.Yellen, Handbook of graph theory, CRC, Press(2004)

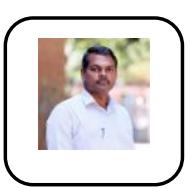

\section{AUTHORS PROFILE}

S.Sriram Assistant Professor, Department of Mathematics, Patrician College of Arts and Science, Adyar,Chennai-20, India.

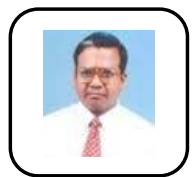

Dr.R.Govindarajan Associate Professor and $\mathrm{Head}$ (Retd.), P.G and U.G, Department of Mathematics, D.G.Vaishnav College, Arumbakkam, Chennai,India. 